Portland State University

PDXScholar

\title{
Sexual Assault Prevention on College Campuses and the Need to Include LGBTQ Students
}

Noor Ayesh

Portland State University

Follow this and additional works at: https://pdxscholar.library.pdx.edu/honorstheses

Let us know how access to this document benefits you.

\section{Recommended Citation}

Ayesh, Noor, "Sexual Assault Prevention on College Campuses and the Need to Include LGBTQ Students" (2017). University Honors Theses. Paper 436.

https://doi.org/10.15760/honors.433

This Thesis is brought to you for free and open access. It has been accepted for inclusion in University Honors Theses by an authorized administrator of PDXScholar. Please contact us if we can make this document more accessible: pdxscholar@pdx.edu. 
Sexual Assault Prevention on College Campuses and the Need to Include LGBTQ Students

By

Noor Ayesh

An undergraduate honors thesis submitted in partial fulfillment of the Requirements for the degree of

Bachelor of Science

In

University Honors

And

Social Work

Thesis Advisor

Dr. Gita Mehrotra

Portland State University 


\section{Background}

Shockingly, one fourth of all female college students are survivors of sexual assault (Burn, 2009). The legal definition of sexual assault is described by the U.S. Department of Justice as any type of sexual contact or behavior without explicit consent of the recipient (U.S. Department of Justice, 2017). Sexual assault on college campuses is one of the main obstacles and a pervasively traumatic event which a student can endure. Statistically, it is now known that women experience sexual violence on college campuses at staggering rates, making them one of the most frequently reported victims (Krakauer, 2015, p.xiv). Community members and survivors of sexual violence have been hard at work attempting to bring societal awareness and prevention in order to reduce the prevalence of sexual assault. However, it still remains that sexual assault on college campuses are being reported at a record high.

One specific example of college campuses taking it upon themselves to address the issue of sexual assault on college campuses is at Missoula State University. Within this college community, author and journalist, Jon Krakauer conducted an investigation of college campus rapes occurring over a four year span. This investigation produced important narratives from college campus sexual assault survivors, tackling common rape misconceptions such as women sending "mixed signals", or other forms of flirtation in order to provoke this heinous crime. Krakauer's work also illustrated the culture of a perpetrator and how perpetrators may get away with sexual violence. Specifically, in Missoula, the discussion focuses on how many perpetrators of sexual assault rely on forgetting the event in order to escape accountability (Krakauer, 2015, p. 211). This is demonstrated in the book by stating how perpetrators can shift the blame, or stating they cannot remember if there was consent. These are just a couple examples of how the 
inability to recollect an event works in the favor of the perpetrator. This relates directly to college campuses accountability for not providing the proper support and prevention on college campuses; where their reports of sexual assault are swept under the rug.

A recent study shows the need for increased support around sexual assault prevention, stating that $25 \%$, of females attending universities are survivors of attempted rape and rape (Burn, 2009; Muehlenhard et al, 2017). As well as the need for college administration to increase awareness of sexual assault occurring to students, but also the need for sexual assault prevention programing and resources on college campuses (Byler \& Miltenberg, 2017). These high rates of sexual assault among females has resulted in a nation wide rise in funding to support sexual assault programming on college campuses; thus, resulting in an increase of funding for Title IX at American universities. Title IX enforces legislation prohibiting discrimination based on sex in federally funded educational programs, tying into sexual assault since sexual assault on college campuses affects females at the highest rates (Shaw, 2016). More importantly, each university is equipped with a Title IX office and a Title IX coordinator, in order to deal with university related issues related to sexual assault (Shaw, 2016). One example of a rise in Title IX funding is found in the 2016 Department of Health and Human Services report, awarded two million dollars to organizations affiliated with college campuses in their efforts to prevent sexual assault on their college campuses (Health and Human Services, 2016). Not only can prevention increases be seen monetarily, but the increase in prevention efforts can also be seen through programs or resources placed on all major college campuses for students. This increase in University awareness of sexual assault can also be seen in the rise in advocates on the Portland State University campus, and the rise in Women's Resource Centers at Universities, in general 
(Howard, 2015). In addition, many Universities have implemented awareness programs, as well as participating in sexual assault awareness month, held in April of every year. For example, Portland State University (PSU) provides many events throughout the month of April. Each of these events provide survivors of sexual assault a safe and inviting space by bringing awareness to this prevalent topic, as well as providing prevention support.

Despite the increase in sexual assault prevention programs on college campuses in recent years, most often there are only one representation of sexual violence in prevention programs that of male violence against women. This framework leaves out the LGBTQ community, a huge community with high rates of sexual assault, for receiving support, services, and awareness (Todahl et al., 2009). However, it is still essential to sexual violence prevention to be aware of and acknowledge that male on female sexual violence is a huge societal issue that continuously needs to be addressed. Based on the high rates of male violence against women, much of the rhetoric and programming around sexual assault prevention and intervention focuses only on male perpetrators, and female heterosexual victims or survivors. The purpose of this analysis and recommendation thesis is to provide more inclusive models and services that include LGBTQ people who experience sexual violence. Most analyses of sexual violence specifically focus on the power and control that perpetrators are looking for; which is commonly expressed in our society through a male taking advantage of and taking away one's females power by sexually violating them. However, this power differential can also be expressed in non-heterosexual interactions and/or by people who have a range of gender identities, and should be addressed in college sexual assault prevention models. 
The lack of inclusion of LGBTQ survivors of sexual assault can be strongly correlated to the old definition of sexual assault and/or rape. Prior to 2012, the legal definition of rape was limited to forcible entry of a penis into the vagina (Menning \& Holtz, 2014). This leaves out the ability for survivors to feel heard and believed, especially if the trauma that happened to the person does not fit a legal definition. This also is believed to affect the report rates of LGBTQ sexual assaults; leading us to believe that the rates of sexual assault amongst LGBTQ people could be higher. Data reports that $60 \%$ of female sexual assault that occurs goes unreported, leaving us with only $40 \%$ of actual reported sexual assaults (Smothers \& Smothers, 2011). These statistics show that women are not reporting sexual violence that happens to them, and this has to due heavily with the societal misconceptions about sexual violence and victim blaming. The high proportion of women underreporting sexual violence makes it difficult for society to fully understand the extend of this issue, leaving the female community even more vulnerable. This can be related to the LGBTQ community, who also experiences similar systemic oppression as the heterosexual women. Since, female victims of sexual violence are not reporting sexual violence that occurs to them, we can assume that other marginalized communities must also be inhibited in their ability to be report the violence the experience and supported in the community.

\section{Sexual Violence in LGBTQ Communities}

Despite women being the highest reported people affected by sexual violence, the LGBTQ community also experiences sexual violence at alarming rates. Overall, one in every ten male identified gay or bisexual male are survivors or victims of rape (Edwards et al, 2015). One particular community within the LGBTQ are lesbians, who experience sexual violence at very alarming rates and are 2 times as likely, than heterosexual females to experience sexual violence 
(Edwards et al, 2015). Despite these high rates, society is not made aware of this through any news reports about the epidemic of sexual assaults; especially since the societal focus is on heterosexual females. One study in 2009 explored what the community understanding of sexual violence towards LGBTQ people was. It concluded that $72 \%$ of the community saw a need for sexual prevention that is tailored to LGBTQ students, but are unaware of where to begin this prevention process (Todahl et al, 2009).

Not only is the community speaking out about a need for inclusive sexual assault prevention, but students who have experienced sexual violence on campuses are speaking out. One way a student's voice is heard is through research conducted at universities. A recent survey conducted at Portland State University (PSU) showed a need for inclusive sexual assault prevention. When a statistical report came out about sexual misconduct on Portland State campus, sadly, $29 \%$ of trans and nonbinary students reported experiencing sexual violence while they were at PSU, and $36 \%$ of all students who experienced sexual violence on campuses reported that their perpetrator was a PSU student (Portland State University, 2017). Not only does this tell the community of the reality that LGBTQ students face when they attend universities, but it also shows a need for administration and students to take a deeper look at the way we see prevention for sexual violence and how we make prevention models on campuses more supportive of all students' experiences.

While the roots for most sexual assault prevention focus on male heterosexual survivors, we must also alter these models to be more inclusive to members of the Lesbian, Gay, Bisexual, Transgender, and Queer Community. Until we begin to include all survivors in college prevention efforts, we will never be able to decrease the amount of sexual violence that occurs to 
our college students. To begin this process, we need to look at current tactics of prevention on college campuses and universities and assess if they can effectively meet the needs of all survivors and communities.

The purpose of this thesis will be to analyze three prevention models and programs commonly implemented on college campuses; specifically to evaluate their responsiveness to the needs of LGBTQ communities. The three models that will be explored are: the Male Peer Support model, the Bystander Intervention Model, and one of the newest models- the Self Defense/Prevention Tactic Model. Each of these models outlined in this thesis are imperative for all communities to understand, primarily for two reasons. The first and foremost reason is that each of these models are supported by positive results in reducing prevention data. Also, these prevention models and programs tie in current important paradigms involving the sexual violence; providing us with a template to begin to add on recommendations in order to better support the LGBTQ people who experience sexual violence. An example of this was found in a research study, conducted with the subjects who participated in the Male Peer Support model; each reported having changes in views around sexual violence (Abbey \& McAuslan, 1998). These changes included male participants reporting that they now feel aware of the contributors to sexual violence, and how unhealthy peer influences can contribute to accepted aggressive behavior, like sexual violence (Abbey \& McAuslan, 1998).

The first model, The Male Peer Support model, addresses harmful masculine tendencies that are promoted by male friendships, which support sexual violence against women. For example, discussing homophobia, and unhealthy masculine friendships and relationships that perpetuate and support sexual assault (Schwatrz \& Dekeseredy, 2001). The second model is the 
Bystander Intervention Model, which was traditionally intended to decrease microaggressions, racism, and other oppressive talk that members of a community encounter; now being used as a model for intervening in sexual assault. (McManon \& Banyard, 2012). The final model which will be analyzed is a fairly new prevention tactic that college campuses are implementing, using self defense as a prevention tactic. This model is directly related to one of the main myths behind sexual assault; where the person being assaulted can and should fight off the person assaulting them. However, this assumption is harmful because it leaves out the ability to understand multiple survival tactics that people use when they are attacked. The analysis of these three models will review these approaches with an eye to LGBTQ inclusiveness. This will then result in a set of recommendations for college campuses to implement into their prevention programs in order to more effectively include and support LGBTQ communities.

\section{Male Peer Support Model}

There are many topics within sexual violence that are not commonly discussed that are essential to the process of being able to prevent sexual violence. The Male Peer Support model, focuses on motivation of perpetrators early on in Male Peer Support process. For example, this Male Peer Support model states that if we focus on promoting healthy male peer relationships, it will will decrease homophobia, and the potential for abusive relationships (Schwartz \& Dekeserdy, 2001). The reasoning behind universities using the Male Peer Support Model, is that it is based on the theory that the narrow conception of masculinity is strongly related to homophobia, conformity, and sexual objectification of women (Schwartz \& DeKeseredy, 2001). These narrow conceptions of masculinity cause unhealthy peer relationships, which can lead to the acceptance of violent behavior. In order to tackle this aspect of sexual assault, the Male Peer 
Support model uses social support theory (Schwartz \& DeKeseredy, 2001). The social support theory assumes that having positive social networks is crucial to maintaining an increased sense of well-being, which then helps people avoid negative experiences (Schwartz \& DeKeseredy, 2001) In addition, developers of this model stated that current male support resources encourage and justify physical, psychological and sexual abuse (Schwartz \& DeKeseredy, 2001). Further showing the community need to pay attention to male resources, and tailor them towards healthy masculine tendencies. If male resources are ignored yet also how this may pull away from the initial goal of prevention.

The next aspect of sexual assault that the Male Peer Support model focuses on is the idea of predatory behavior within male per relationships. This strong focus on predatory sexual conduct, shedding light on the benefit in looking at those who are predators of sexual misconduct, and how we can interfere in this behavior (Alvi et al, 2001). Initially when hearing about the high rates of sexual violence on college campuses, the community is left with the following question: How can such a low crime rates environment, like a college campus, also become a high crime rate area for sexual violence? The Male Peer Support model tackles on that question, by tying in male peer relationships to the rise of sexual assault on college campuses. Developers of this model believed that men are involved in more unhealthy peer relationships than compared to women, which is believed to lead more males to perpetrating physical violence and/or sexual violence towards women (Schwatrz \& Dekeseredy, 2001). The primary focus of this model is the idea that men develop compulsive masculine behaviors, and are not inherently born with them. Leading away from the "boys will be boys" notion, by replacing it with the idea that these habits are learned and not the inherent nature of masculinity. 
This prevention model was developed to mainly be taken into fraternities, on the basis that it is believed that fraternity members showed more aggressive male peer relations; relating to the theory that many students involved in athletic clubs or fraternities have a narrow conception of masculinity, causing harmful masculine rhetoric and behavior. One way that the Male Peer Support model stands out is because it is one of the only prevention models that includes motivation, as a key to prevention. Meaning that, a clear understanding of motivations behind sexual violence is beneficial in producing prevention models. Specifically, attempting to find where this motivation for sexual violence in male dominated peer groups is so rampant. This motivation can be rooted in homophobia, sexism, or racism. Including these biases in prevention models is important in working towards including all survivors.

This model also produced the data that male undergraduates encourage assault towards their male peer partners, reiterating that masculinity can promote abuse (Alvi et al, 2001). This model includes an understanding for prevention by going into the depth; their three key factors to sexual assault; Showing the key factors to sexual violence being the presence of likely offenders, absence of affective guardianships and availability of suitable targets (Alvi et al, 2001). This is to say that the presence of likely offenders means that there is the presence of people who have the potential to sexually harm someone. The absence of affective guardianships is when a potential target of sexual violence does not have people around them to interfere if something were to go wrong. The model uses the term suitable targets to mean intoxicated people, since many perpetrators use alcohol in order to impair their victims judgments (Schwatrz \& Dekeseredy, 2001). 
These presence of key factors are common in a college party environment. For example, the presence of likely offenders rises due to the fraternity and male dominated organizations' narrow conception of masculinity and increase violence towards women. In addition, the lack of healthy guardianship, is explained as peer groups for this context. The absence of affective guardianships, providing the solution that male peers can provide that guardianships within each other (Alvi et al, 2001). Unfortunately, party environments can bring on what the model referred to as, "suitable targets". The models explains that suitable targets, are caused by increased drinking and/or drug use, implying that a suitable target for sexual assault is one who is intoxicated or impaired. Since, their is an increase in the suitable targets in a college party, this calls for a need of effective guardianship. And since formal parental guardianships cannot be achieved in a college party space, this model suggests that this guardianship should be done by the community, specifically the men on campus (Alvi et al, 2001).

The Male Peer Support Model is then broken down into parts to explain how this intervention model should be used. Specifically talking about the social support theory again, this explains that the actions of others have; primarily, the main effects and direct effects on the people around them. This model focuses on encouraging men to have increased social networking help in order to avoid negative experiences by choosing more positive peer groups. The model then goes on to explain the buffering stage, used for those who are under current stress. This step instead of showing a person the negative experiences, it instead protects them directly from these negative experiences, or buffers them from the wrong type of social support. The current model focuses on reconstructing the male peer relationships in order to lower rates of sexual assault, and provide men with healthy constructive male friendships. However, this 
model has been modified, to be effective with a student population; in order to help prevent sexual assault.

Another important finding in the Male Peer Support model is that men with high levels of life events or stress had or have abusive peers which related to abuse against women (Schwartz \& Dekeserdy, 2001). This shows that there is a need for positive peer support services for men, to begin to change the harmful connection between masculinity and sexual violence against women. around sex. By doing so, this will enhance the men's ability to discuss topics like sexual violence with other men. This ability to discuss these topics will open up the space to questioning violence, and in doing so, will open up the awareness that sexual violence can affect people outside of the female heterosexual community.

\section{Bystander Intervention}

Another sexual assault prevention model is the Bystander Intervention model. This model provides scenarios and information of how the bystanders should act in accordance to oppressive events; especially when sexual violence are taking place. This model focuses on preparing college students to intervene if they believe sexual assault or violence may occur (McManon \& Banyard, 2012). In this model, students are presented with with scenarios to practice the templates of intervention strategies, in order to confront victim blaming or other talk that may perpetuate sexual violence (McManon \& Banyard, 2012)

Bystander Intervention is a potential tool for sexual assault prevention, shifting the focus of sexual assault prevention to peers and community members. Beginning in 1970, Latane and Darley produced a five step prevention model, in response to the theory that when people are around others, they are less likely to help, than when they are alone. Furthermore, this makes 
college culture a frightening experience. Unfortunately, there is not safety in numbers and we must take it upon the community in order to keep each other safe. Many researchers have modified the Latane and Darley's model in order to help vulnerable populations. Shawn Burn specifically used the model, and conducted a preliminary test to determine if there is a correlation between Bystander Intervention and the potential to decrease sexual violence (2009).

The Bystander Intervention model has been modified in order to fit the criteria needed to access preventing sexual violence. This model was produced using the theory that when we are groups, we are individually less likely to offer help when it is needed (Pugh et al., 2016). The reason for this is because we rely on the other people around us to intervene. This can be incredibly detrimental in a community setting, like a college party. By leaving students with the interpretation that there is not even safety in numbers.

Burn (2009) used Bystander Intervention as a foundation to tackle the immense amount of rape culture and rape myths in our society. Burn's study identified the main barriers that will need to be addressed in order to make Bystander Intervention effective in preventing sexual violence. These barriers included the perpetuation of sexual violence, from rape culture and rape myths (Pugh et al., 2016). Rape culture is acceptable practices within a society or culture that promotes and normalizes sexual violence. Rape myths are the false attitude society has about rape and the willingness to assign the blame to sexual assault survivors. These toxic cultural norms need to addressed with college students, before we can properly attempt to prevent sexual assault or violence. For example, a common occurrence on college campuses is when no one intervenes on a sober man, taking an overly intoxicated woman home; not seen as a potential sexual assault situation. This inability in willingness for others to help in this situation is strongly 
related to the lack of understanding of what sexual assault or violence looks like. Thus, this model focuses on building awareness to what sexual violence can look like, as well as, how to intervene if it may be happening.

In order to address sexual violence prevention, Burn (2009) kept the initial five steps to helping, that was derived from the regional model. These steps to intervention or to helping are as follows. Step one is noticing the event is occurring. This step provides recommendations students to be aware of no to no the safety of themselves, but others around them. The next step is to acknowledge that an intervention is necessary. Is it important to be aware that these two steps are hard to achieve, especially in a college environment. This was brought up in Burn's research, when many participants state that they are unable to determine if the situation needed intervening (Burn, 2009). Furthermore, proving prevention models like these are needed on college campuses; in order to squash rape myths and rape culture. The next step is accepting responsibility to help, followed by making a plan on how to act. The final step is actually intervening in the situation (Burn, 2009). It is in these final two steps, [the plan of action and actually intervening], where students are able to discuss when they should intervene and practice ways of intervening in order to prevent someone from a potential sexually violent situation.

When initially thinking of these five steps, we can presume that these should be easily achieved. However, there is more to this prevention model than just supplying students with these five steps. Instead, this model is intended to equip students with the tools they need to prevent sexual violence through the use of interactive scenarios. Of course, these scenarios are common situations seen in college environments; essentially for students to be able to apply 
these tools to events they most likely will come across. This model is heavily based on the idea that the main resolution to sexual assault and violence prevention is reliant on our community.

\section{Self-Defense as Sexual Assault Prevention}

Another prevention tactic to sexual assault is self defense; much like Bystander Intervention, also provides the community with reactions to particular events. This last model, Feminist Self-Defense and Resistance Training is very different from the first two models. This model, in itself, is a proposal for college campuses to support potential victims in using self defense as a way to prevent the occurrence of sexual assault. Self defense, alone, has been used commonly in order to provide women with the ability to defend themselves physically; if they were to be in a violent situation. This tactic for prevention of sexual assault is commonly offered only through martial arts agencies, or law enforcement (Dardis \& Gidycz, 2014). However, just using self defense on its own has been critiqued due to the limitation it places on women's freedom and the potential it has to foster fear that they could be attacked at anytime (Dardis. \& Gidycz, 2014). Which leads into how Feminist Self-Defense and Resistance Training was produced, based on criticisms of already existing self defense programs. Feminist approaches focus on empowering women to focus on techniques to help them feel powerful and strong (Dardis \& Gidycz, 2014). In order to empower women, this prevention model ties in the reality of sexual violence by focusing on the historical effect of female" niceness", which promotes women to be passive (Dardis \& Gidycz, 2014). Additionally, an open dialogue gives women the power to feel strong and able to defend themselves, and is one way to tackle on multiple components of sexual violence prevention.

This proposed model gives female participants the skills they need to physically defend 
themselves and also gives recommendations for verbal language to deter sexual assault (Dardis \& Gidycz. 2014). This model also provides recommendation to women to listen to the voice within (aka intuition), in order to help them see a situation that may end badly; focusing on the verbal and nonverbal techniques women can use to attempt to provide a safe space for themselves (Dekeseredy, 2014). This models additionally focuses on the fact that colleges or the “ivory tower" are one of the top places where sexual assault happens.(Dekeseredy, 2014). The use of the term "ivory tower" symbolizes that a college campus appears to be a safe and beautiful place. However, when we peel back the layers, we discover a culture that normalizes and justifies sexual violence. This model is geared towards female victims of sexual assault and touches on an important topic that the previous models did not specifically address - the paradigm of how gender socialization and inequalities impact the way women can react to sexual violence.

Self defense, as a sexual assault prevention model, can be seen as both positive and negative to a college climate. Self defense classes, specifically for women, allow one community who is deeply affected by sexual violence with a space to comfortably practice how to defend themselves. Yet, it is very important to continue to have female specific self defense classes, since physical defense is one way to avoid a sexually violent situation. Also having a female-specific class can provide a safer space for females to practice their physical strength in a non-judgemental and open environment. Nonetheless, only focusing on physical defense implies that the victim is in charge of solely getting out of the situation, and perpetuates the myth that if she experiences violence it is because the victim did not fight hard enough. At any rate, people are using the backing of feminism to implement self defense as a need for all students. 
In addition to self defense, there has been research attempting to understand females responses to characteristics of rape stimuli (Anderson et al, 2015). One qualitative study used audio footage to record females responses to the potential of acquaintance rape (Anderson \& Cahill, 2014). This is important in the context of self defense prevention models in colleges, since sexual violence perpetrators typically know this victims. In order to support students to properly physically defend themselves against sexual violence, we must first understand what boundaries they may encounter when presented with the scenario. In this study, the findings conclude that many women's first response to the threat of sexual violence is to verbally communicate boundaries towards their potential perpetrator, and the last common response was physical assertion (Anderson et al, 2015). This implementation of self defense courses is important in the aspect of prevention, because this prevention tactic works to help women understand one of the responses they may have to sexual violence.

Traditionally, many women do not have the knowledge of ways to physically protect themselves. And, when they enter into college, having this knowledge of protection can be favorable. This prevention model could be especially useful if it was implemented for students during their freshmen year where they are at higher odds of experiencing sexual violence (Dekeseredy, 2014). It is also important to understand that self defense being used as a prevention model for sexual violence assumes that women will find the situation threatening enough that it warrants physical violence (Anderson et al, 2015). This is harmful, as it assumes that the community has the same definition of sexual violence and will be able to react to these situations in order to keep themselves safe. Instead, self defense sexual assault prevention 
models need to implement scenarios that display the wide range of sexual violence potential sexual violent situations. This can range from common college rape jokes to microaggressions

\section{Analysis of the Models}

In order to determine if these sexual assault prevention models are inclusive of LGBTQ students who experience sexual violence, these models were looked at through a critical lens; beginning with analyzing these models and identifying specific themes throughout the literature on each particular model to determine LGBTQ inclusion. In doing this, the analysis focused on how the models used gendered language, and how the use of binary conceptualizations of gender impacts the ability to include all people affected by sexual violence. The other code was the mention of sexuality - specifically paying attention to the language mentioning sexuality. And, if there was not, having an better understanding of what the implied sexuality behind the sexual assault prevention models. Finally, a review of the stories or scenarios that were included in the prevention models to see if any of them included LGBTQ people or scenarios. By deciding to look directly at the work, usage, and intention behind how college campuses look at sexual violence, this enables the community to potentially have a better understanding of the challenge and unique experiences LGBTQ students face when on a college campus. Critically analyzing the way universities have used language to prevent sexual assault will also contribute to the recommendation section, to be able to break down boundaries to supporting LGBTQ students.

Currently, there is little research and literature about sexual violence within the LGBTQ community. However, as noted above, this does not mean that sexual violence is not present within the LGBTQ community. We can see that through PSU statistics mentioned in the background section of this thesis, the minimal existing research, and also in a groundbreaking 
anthology, Queering Sexual Violence, published in 2016. By compiling the existing literature about sexual violence in the LGBTQ community and Queering Sexual Violence, the following recommendations should be made, in order to support LGBTQ students on college campuses who face sexual violence. Given that there are very few resources available that relate directly to LGBTQ people expriencing sexual violence, Queering Sexual Violence is an important tool for universities. Queering Sexual Violence does not focus on only one aspect of sexual violence, but multiple aspects of the issues facing LGBTQ survivors. For example, one part of the book focuses on how the use of specific language can be exclusive of the LGBTQ community on a college campuses. Specifically, the use of survivor hood and victimhood; as a primary language usage that does not highlight all peoples experiences with sexual violence (Patterson, 2016). This text also provides a variety of narratives directly by people in the LGBTQ community who have experienced sexual violence, providing more of a framework for colleges to use in implementing inclusive sexual assault prevention (Reza, 2017).

In reviewing the three prevention models outlined above, it was found that these models did not have a variety of narratives about sexual violence, but instead primarily highlighted females who experienced sexual violence from men. This was true in the Male Peer Support model, as well as the Bystander Intervention model. The stories that were used as scenarios, were usually party situations; where harmful masculinity is displayed towards a vulnerable young female student. Yet statistically, those within the LGBTQ community are also affected by sexual violence at high rates.

Another noticeable flaw in the prevention models that inhibits LGBTQ students from being included, is the use of gendered language and the assumption of the gender binary. The 
gender binary refers to the societal notion that gender can only be male or female. However, it is possible for gender to neither be male nor female (Newman, 2002). The gender binary leaves out people in the LGBTQ community; specifically intersex, non-binary and trans people's. Yet, in spite of this, the gender binary was very prevalent in the prevention models analyzed. For example, The Male Peer Support Model was specifically focused on, with the notion that only masculinity can be harmful enough to be a factor for sexual violence. By only focusing on one gender for being the sole perpetrator, this excludes students who may identify differently or have a different definition of gender. This Male Peer Support Model is an example of how specific gendered language can assume the gender binary, and can leave out students in the LGBTQ community who have a broader definition of gender.

\section{Recommendations}

In order to make sexual assault prevention programs on college campuses more inclusive to LGBTQ communities, programs need to be modified and developed to meet the unique needs of these communities. One important recommendation to including LGBTQ in campus prevention is to change language used in prevention models. Despite it being important to have resources for male peer relationships, like mentioned in the Male Peer Support model, we must also a variety of spaces for students of diverse gender identities to go to. For example, having trans-specific refuge space or sanctuaries: since the trans community experiences violence at higher rates than compared to other parts of the LGBTQ community. Statistically, on Portland State Campus, $29 \%$ of trans and nonbinary students experience sexual violence, and $4 \%$ of men experience sexual violence (Sexual Misconduct Campus Climate Survey, 2016). These statistics 
are brought up again here in order to demonstrate that while there is a need for male peer spaces, there also needs to be safe spaces for trans and nonbinary students.

The Bystander Intervention model (and self-defense training) is a prevention model that is not specific to either a particular sexual orientation or for a specific gender, (such as in the Male Peer Support Model). Thus, the Bystander Intervention Model is a good framework to begin to see how we can implement scenarios into students education; to better understand how to prepare them to intervene in situations that can lead to sexual violence. Relative to this, one main critique to sexual violence prevention is that the studies around sexual violence assume heterosexuality (Edwards et al, 2015). This blatant assumption in studies also transfers over to sexual assault prevention by only providing prevention stories about male on female sexual violence; leaving out a community affected by sexual violence. Adding more scenarios that reflect LGBTQ experiences of violence can help expand understandings of sexual violence that are more inclusive of these communities.

Another way we can change the language in sexual assault prevention approaches, is to look at alternative language to the words survivor and victim. This intended visual of a survivor leaves a person who has experienced sexual violence, but does not identify as a survivor with the perception of those who are struggling to manage the healing process after sexual trauma. This harmful rhetoric of survivor versus victim was brought up in Queering Sexual Violence, shedding light on the need to change language used in sexual violence prevention. This was brought up based on research from Rousse Arielle, who conducted a small survey with LGBTQ members from different backgrounds, asking what words they associated with either victim or survivor (Arielle, 2016). The responses yielded very negative words like weak and damaged 
with victim, and powerful and speaking out were associated with survivor (Arielle, 2016). Based off the systematic oppression and persecution of the LGBTQ community, respondents mentioned that it was very difficult for themselves to identify as a survivor because they did not feel all the words associated with it; based off their oppressed position within society (Arielle, 2016). This shows that there is a need to expand the language we use in anti-sexual violence prevention, but also be aware that the current language may not be as inclusive as believed.

In order to support students, we need to highlight those who do not meet the predictable or expected path towards healing from sexual trauma (Patterson, 2016). For instance, survivor seems to be a very inclusive term. Yet, by only allowing people to identify with either victim or survivor, this leaves out the voices of millions of people who have survived systematic oppression and historical trauma (Patterson, 2016). Survivor hood was created in spite of victim, which then causes the term survivor to be incredibly harmful and oppressive towards those to have experienced sexual trauma. When we hear stories about sexual assault on college campuses, we are most often met with images of the vulnerable, middle class, heterosexual female who is now fighting back against sexual violence. Arguably, this leaves out communities whose voices are already silenced in society. LGBTQ voices are not often included or heard when conversations about sexual violence occurs. Instead, the societal focus is almost exclusively on maintaining a safe space for heterosexual females, instead of all individuals affected by sexual trauma on college campuses.

Not only do we need to change the language, but we also need to have exposure to more than one sexual violence story on college campuses. When discussing those affected by sexual violence, we need to include narratives that all students can identify with. Queering Sexual 
Violence is one resource college campuses can use to help prevent sexual violence in the LGBTQ community on college campuses. This text includes many stories from LGBTQ people who have experienced sexual violence. This points to a primary recommendation to utilize an inclusive lens and language for all individuals, including all members of the LGBTQ community who are victims of sexual assault.; thus, moving away from prevention models that only mention the act from a heterosexual male perpetration towards a heterosexual female victim. The main reason having a variety of narratives of sexual violence for students to be aware of is because this allows students to look past the societal misconception that sexual violence is only something that occurs to women, by heterosexual men. Also, since multiple communities (Men, Women and LGBTQ) are affected by the same violence, both narratives should used in training scenario situations in order to prepare students to intervene and prevent sexual violence. If students are only equipped with one prevention scenario, students will only see sexual violence through one lens; leaving out the awareness of sexual violence in the LGBTQ community.

In relation to the above recommendation, another way to include all students affected by sexual violence, is to expand safe spaces on college campuses. In order to include students in sexual assault prevention, we need to have spaces where they can meet and discuss these topics. This recommendation is not for one type of safe space, but instead for a variation in the types of safe spaces. Safe spaces were intended to provide a place for students to express their feelings and break down barriers. However, these safe spaces may not be as inclusive as we think. For instance, the self defense training offered are typically safe spaces for women to learn how to defend themselves against perpetrators. This idea that only women need to learn self defense 
further excludes those in the LGBTQ community, because this separation of male and female implies we all have the same definition of those genders (Patterson, 2016). Because of this divide in safe spaces, many trans students have reported feeling unwelcome or unsafe walking into some safe spaces; specifically using language being used for "women" or Gay/Straight Alliances, which may inadvertently leave out trans, bisexual and other sexual and gender minority students from these spaces (Wagner. 2014). Instead, of focusing on one type of safe spaces, we need to shift the thinking to include conversations, programs, and spaces that are attentive to the range of gender and sexual orientations and identities (Patterson, 2016).

\section{Conclusion}

A college campus should not be a place where students are at a higher risk for sexual violence. Instead, inclusive and safe prevention and education should be the main priority, a in order to provide a safe environment for students prevention of sexual violence, the main focus should be on the university administration. One way to show students that the administration is on their side, is to show support by expanding their sexual violence prevention education; bridging a gap with a community that deeply needs support. Not only does the PSU sexual misconduct climate survey (2017) show that LGBTQ students needs support around sexual violence, but the lack of research around sexual violence shows that this community is being swept under the rug. Instead, PSU and all Universities should hold to their standards of inclusion, and work towards preventing sexual violence on campus. Universities can attempt to prevent sexual violence by increasing behavioral skills and training for staff, in order to equip them with the ability to intervene on potentially sexually violent, or other forms of violent situations. PSU and most University campuses have resources for queer and trans students already established, 
therefore it should be given that PSU work collaboratively with these groups to expand their sexual assault prevention tactics to reach the LGBTQ student population. By including stories, prevention models and safe spaces that demonstrate ideas outside of assumed heterosexuality and gender binary status, this will allow students to expand their understanding of sexual violence, and furthermore be able to intervene in situations where LGBTQ students may be at more risk for sexual violence. Furthermore, in the current political climate of America, it is even more crucial that we support LGBTQ students and create an acute awareness to the multiple communities of students affected by sexual violence. It is a hard task to take on, to change the mindsets of others, and the diligent work necessary in preventing further sexual violence. However, a college setting is the perfect place to begin challenging one's current beliefs; towards promoting a more equitable and safe space to access a higher education. By critically looking at current campus sexual assault prevention models and using these current models as a foundation to provide more inclusive prevention sexual assault models and resources for LGBTQ students, universities can begin to to tailor them to address all communities affected by sexual violence.

It is important to understand that when discussing sexual violence, those directly affected by it are the only ones who have the ability to speak for their experience and label themselves either a victim or survivor. Both of these labels have heavy weight and shame tied to them due to society, and this thesis is looking to decrease the shame and increase the awareness and support for all people affected by sexual assault and violence. There are many barriers, including stigma, that make it very difficult for students on a college campus to feel safe and supported. The Male Peer Support Model and Bystander Intervention both touch on this need for community 
togetherness. however, to promote sexual violence prevention, it would be imperative to incorporate narratives within Feminist Self-Defense and Resistance Training, and for college campus administrators to adjust sexual assault prevention programs to include LGBTQ people, which will allow for more positive college experiences. Not only do we need to make adjustments to existing prevention models, but we need to band together as a community for this resolution. 


\section{References}

Abbey, A., \& McAuslan, P. (1998). Sexual assault on the college campus: The role of male peer support/women abuse on campus: Results from the canadian national survey. Sex Roles, 39(3-4), 330-332.

Arielle, R. (2016). Queering sexual violence. Beyond the binaries: Exclusive dichotomies in the anti-sexual violence movement. Riverdale Avenue Books.

Burn, S. (2009). A Situational model of sexual assault prevention through bystander intervention. Sex Roles, 60(11-12), 779-792. doi:10.1007/s11199-008-9581-5

DeKeseredy, W. S., Tait, D., \& Alvi, S. (2001). Male peer support and a feminist routine activities theory: Understanding sexual assault on the college campus. JQ: Justice Quarterly, 18(3), 623.

DeKeseredy, W. S. (2014). Thinking Critically About Campus-Based Self-Defense Programs: A Response to Christine Gidycz. Trauma, Violence \& Abuse, 15(4), 334-338. doi:10.1177/1524838014521024

Department of Justice (2016) Sexual assault. Retrieved from https://www.justice.gov/ovw/sexual-assault

Edwards, K. M., Sylaska, K. M., Barry, J. E., Moynihan, M. M., Banyard, V. L., Cohn, E. S., \& .Ward, S. K. (2015). Physical dating violence, sexual violence, and unwanted pursuit victimization: A Comparison of incidence rates among sexual-minority and heterosexual college students. Journal Of Interpersonal Violence, 30(4), 580-600. 
doi:10.1177/0886260514535260

Howard, B. (2015). How colleges are battling sexual violence. US and World Report News. Retrieved from https://www.usnews.com/news/articles/2015/08/28/how-colleges-are -battling-sexual-violence

Krakauer, J. (2016). Missoula. Random House USA.

McMahon, S., \& Banyard, V. L. (2012). When can i help? A conceptual framework for the prevention of sexual violence through bystander intervention. Trauma, Violence \& Abuse, 13(1), 3-14. doi:10.1177/1524838011426015

Menning, C. L., \& Holtzman, M. (2014). Processes and patterns in gay, lesbian, and bisexual sexual assault: A Multimethodological sssessment. Journal Of Interpersonal Violence, 29(6), 1071-1093. doi:10.1177/0886260513506056

Miltenberg, A. T., \& Byler, P. A. (2017). College sexual misconduct disciplinary proceedings. Gpsolo, 34(2), 62-63

Muehlenhard, C. L., Peterson, Z. D., Humphreys, T. P., \& Jozkowski, K. N. (2017). Evaluating the one-in-five statistic: Women's risk of sexual assault while in college. Journal of Sex Research, 54549-576. doi:10.1080/00224499.2017.1295014

Newman, L. K. (2002). Sex, gender and culture: Issues in the definition, sssessment and treatment of gender identity disorder. Clinical Child Psychology \& Psychiatry, 7(3), 352.

Patterson, J. (2016). Queering sexual violence. Riverdale Avenue Books

Portland State University. Sexual Misconduct Campus Climate Survey. (2016). Retrieved from 
https://www.pdx.edu/diversity/sexual-misconduct-campus-climate-survey

Pugh, B., Ningard, H., Ven, T. V., \& Butler, L. (2016). Victim ambiguity: Bystander Intervention and dexual assault in the college drinking scene. Deviant Behavior, 37(4), 401-418. doi:10.1080/01639625.2015.1026777

OASH. (2016). HHS award \$6 million to prevent sexual assault on college campuses. Health and Human Services. Retrieved from https://www.hhs.gov/ash/about-ash/news/2016/ Hhs-awards-6m-prevent-sexual-assault-college-campuses.html

Reza, S. (2017). Queering sexual violence: Radical voices from within the anti-violence movement. Bitch Magazine: Feminist Response To Pop Culture, (75), 67-68.

Schwartz, M. D., Smothers, M. K., \& Smothers, D. B. (2011). A sexual assault primary prevention model with diverse urban youth. Journal Of Child Sexual Abuse, 20(6), 708-727. doi:10.1080/10538712.2011.622355

Shaw, L. E. (2016). Title IX, sexual assault, and the issue of effective consent: Blurred lines--when should "yes" mean "no"?. Indiana Law Journal, 91(4), 1363-1423.

Steck, A. K., \& Perry, D. R. (2016). Fostering safe and inclusive spaces for LGBTQ students: Phenomenographic exploration of high school administrators' perceptions about GSAs. Journal Of LGBT Youth, 13(4), 352-377. doi:10.1080/19361653.2016.1185759

Todahl, J. L., Linville, D., Bustin, A., Wheeler, J., \& Gau, J. (2009). Sexual assault support services and community systems. Violence Against Women, 15(8), 952-976.

Wagner, L. (2014). Creating safer spaces for LGBTQ students. Leadership, 43(5), 18-20 